\title{
Synthesis of novel alkaloid derivatives from the vinyl ether of lupinine and PH-compounds
}

\author{
Nina K. Gusarova, ${ }^{a}$ Svetlana F. Malysheva, ${ }^{a}$ Ludmila A. Oparina, ${ }^{a}$ Nataliya A. \\ Belogorlova, ${ }^{a}$ Anatolii P. Tantsyrev, ${ }^{a}$ Lidiya N. Parshina, ${ }^{a}$ Boris G. Sukhov, ${ }^{a}$ Rustam \\ T. Tlegenov, ${ }^{b}$ and Boris A. Trofimov ${ }^{a_{*}}$ \\ ${ }^{a}$ A.E. Favorsky Irkutsk Institute of Chemistry, Siberian Branch of the Russian Academy \\ of Sciences, 1 Favorsky St., Irkutsk 664033, Russia \\ ${ }^{b}$ Karakalpakstan State University, 1 akad. Abdirova Street, Nukus 742012, Karakalpak \\ Republic, Uzbekistan \\ E-mail: gusarova@irioch.irk.ru
}

\begin{abstract}
A new family of functional quinolizidine alkaloids containing alkoxy groups and phosphine or phosphine chalcogenide fragments has been prepared from the vinyl ether of enantiomerically pure lupinine, secondary phosphines and phosphine sulfide. The reaction proceeds readily under radical initiation to give anti-Markovnikov adducts, dialkyl[2-(octahydro- $\mathrm{H}$-quinolizin-1ylmethyloxy)ethyl]phosphines and -phosphine sulfide, in high yields (89-96\%). The phosphine moieties of the alkaloids synthesized have been oxidized with air, elemental sulfur or selenium to afford the corresponding phosphine chalcogenides.
\end{abstract}

Keywords: Vinyl ether of lupinine, secondary phosphines and phosphine chalcogenides, radical addition, functional quinolizidine alkaloids

\section{Introduction}

Quinolizidine alkaloids are widely distributed in plants. ${ }^{1}$ They possess a diverse range of biological activities, e.g. cardiotonic and myotonic, ${ }^{2}$ anticancer, ${ }^{3}$ anti-HBV, ${ }^{4}$ antimicrobial and antibacterial. ${ }^{5}$ They also affect the nervous system state. ${ }^{6}$ Much attention from researchers is now focused on the study of the biological activity of quinolizidine alkaloid derivatives to design drugs of new generation. ${ }^{7}$ For example, lupinine derivatives are prospective ligands for sigma receptors in the central nervous system, which are implicated in the pathogenesis of psychiatric and motor disorders. ${ }^{8}$ Recent data indicate the octahydroquinolizidine chemotype is the structural motif of derivatives with calcium channel antagonist activity ${ }^{9}$ as well as inhibitors of histone 
deacetylase. ${ }^{10}$ Therefore, the search for convenient methods for the preparation of novel functional quinolizidine alkaloids remains an urgent synthetic challenge.

In this paper we describe an atom-economic synthesis of previously unknown lupinine derivatives from the vinyl ether of lupinine $\mathbf{1}$ and now available secondary phosphines ${ }^{11}$ or phosphine sulfides ${ }^{12}$ readily prepared by the direct reaction of red phosphorus with alkyl halides $^{11 \mathrm{a}}$ or styrene. ${ }^{11 \mathrm{~b}}$ The choice of vinyl ether $\mathbf{1}$ is due to its recently described synthesis by direct vinylation of lupinine with acetylene. ${ }^{13}$ To date, the free radical additions of H-phosphines and H-phosphine chalcogenides to vinyl chalcogenides is just scarcely studied. Only few publications dealing with the radical addition of secondary phosphines and phosphine chalcogenides to vinyl ethers are known. ${ }^{14}$

\section{Results and Discussion}

Secondary phosphines 2, 3 and phosphine sulfide $\mathbf{4}$ add to vinyl ether $\mathbf{1}$ regiospecifically in the presence of azaisobutyronitrile (AIBN) at $65-70{ }^{\circ} \mathrm{C}$ to form the corresponding optically active tertiary dialkyl[2-(octahydro-2H-quinolizin-1-ylmethyloxy)ethyl]phosphines 5, 6 and -phosphine sulfide 7 in high yields (Table 1).

Table 1. Synthesis ${ }^{\mathrm{a}}$ of tertiary phosphines $\mathbf{5 , 6}$ and phosphine sulfide $\mathbf{7}$

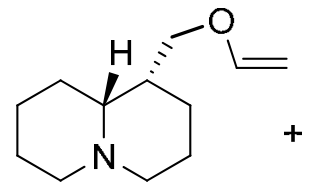

1

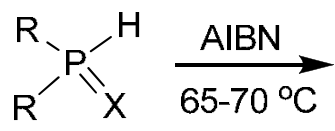

2-4

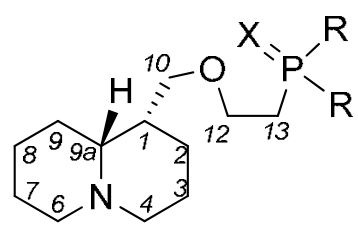

5-7

\begin{tabular}{|c|c|c|c|c|c|c|}
\hline \multirow[t]{2}{*}{ Entry } & \multirow{2}{*}{$\begin{array}{c}\text { Vinyl ether } \mathbf{1} \\
(\mathrm{mmol})\end{array}$} & \multicolumn{3}{|c|}{ PH-reagent $(\mathrm{mmol})$} & \multirow[t]{2}{*}{ Time (h) } & \multirow{2}{*}{$\begin{array}{c}\text { Product } \\
(\text { yield, \% } \%)^{b}\end{array}$} \\
\hline & & & $\mathrm{R}$ & $X$ & & \\
\hline 1 & 2.50 & $2(2.50)$ & $\mathrm{Bu}$ & none & 10 & $5(93)$ \\
\hline 2 & 3.10 & $3(3.10)$ & $\mathrm{Ph}\left(\mathrm{CH}_{2}\right)_{2}$ & none & 168 & $6(89)$ \\
\hline 3 & 1.02 & $4(1.02)$ & $\mathrm{Ph}\left(\mathrm{CH}_{2}\right)_{2}$ & $\mathrm{~S}$ & 4 & $7(96)$ \\
\hline
\end{tabular}

${ }^{a}$ Heating of the reactants at $65-70{ }^{\circ} \mathrm{C}$ in the presence of AIBN $(0.5-1.5 \mathrm{wt} \%$ of the reactants' mass). ${ }^{\mathrm{b}}$ Isolated yields.

Phosphines 5, 6 react smoothly with oxygen, elemental sulfur or selenium in organic solvents to give the corresponding optically active phosphine oxides $\mathbf{8}, \mathbf{9}$, phosphine sulfides $\mathbf{7 , 1 0}$ and phosphine selenide 11 (Table 2). 
Table 2. Synthesis of tertiary phosphine chalcogenides 7-11

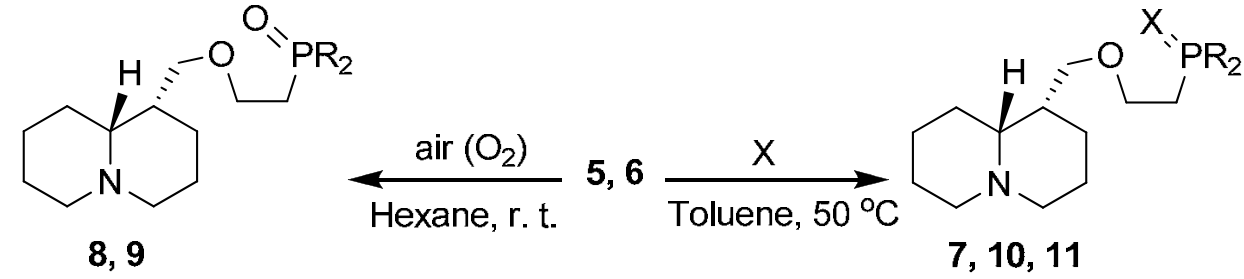

\begin{tabular}{|c|c|c|c|c|c|c|c|}
\hline \multirow[t]{2}{*}{ Entry } & \multicolumn{2}{|c|}{$\begin{array}{c}\text { Tertriary phosphine } \\
(\mathrm{mmol})\end{array}$} & \multirow[t]{2}{*}{$\begin{array}{l}\text { Oxidant } \\
\text { (mmol) }\end{array}$} & \multirow[t]{2}{*}{$\begin{array}{l}\text { Temperature } \\
\left({ }^{\circ} \mathrm{C}\right)\end{array}$} & \multirow[t]{2}{*}{$\begin{array}{l}\text { Time } \\
\text { (h) }\end{array}$} & \multirow[t]{2}{*}{$\begin{array}{l}\text { Solvent } \\
\text { (ml) }\end{array}$} & \multirow[t]{2}{*}{$\begin{array}{c}\text { Product } \\
(\text { yield, \% } \%)^{\mathrm{a}}\end{array}$} \\
\hline & & $\mathrm{R}$ & & & & & \\
\hline 1 & $5(0.44)$ & $\mathrm{Bu}$ & $\begin{array}{l}\text { air }\left(\mathrm{O}_{2}\right), \\
\text { excess }\end{array}$ & $20-22$ & 1 & $\begin{array}{l}\text { Hexane } \\
\text { (2) }\end{array}$ & $8(95)$ \\
\hline 2 & $5(0.88)$ & $\mathrm{Bu}$ & $\begin{array}{l}X=S \\
(0.88)\end{array}$ & 50 & 3 & $\begin{array}{l}\text { Toluene } \\
\text { (4) }\end{array}$ & $10(88)$ \\
\hline 3 & $6(0.34)$ & $\mathrm{Ph}\left(\mathrm{CH}_{2}\right)_{2}$ & $\begin{array}{c}\text { air }\left(\mathrm{O}_{2}\right) \\
\text { excess }\end{array}$ & $20-22$ & 3 & $\begin{array}{l}\text { Hexane } \\
\text { (2) }\end{array}$ & $9(97)$ \\
\hline 4 & $6(0.69)$ & $\mathrm{Ph}\left(\mathrm{CH}_{2}\right)_{2}$ & $\begin{array}{l}X=S \\
(0.80)\end{array}$ & 50 & 3 & $\begin{array}{l}\text { Toluene } \\
\text { (5) }\end{array}$ & $7(90)$ \\
\hline 5 & $6(0.75)$ & $\mathrm{Ph}\left(\mathrm{CH}_{2}\right)_{2}$ & $\begin{array}{l}X=\mathrm{Se} \\
(0.89)\end{array}$ & 50 & 3 & $\begin{array}{l}\text { Toluene } \\
\text { (5) }\end{array}$ & $11(75)$ \\
\hline
\end{tabular}

${ }^{\mathrm{a}}$ Isolated yields.

\section{Conclusions}

A straightforward atom-economic ("green") method for the preparation of previously unknown alkaloid derivatives containing lupinine core, alkoxy groups and phosphine or phosphine chalcogenide fragments, using vinyl ether of enantiomerically pure lupinine, secondary phosphines and phosphine sulfide has been successfully developed. The functional lupinine derivatives synthesized can be widely used for the direct design of biologically active compounds as well as easily accessible enantiomerically pure chelating ligands for preparing transition metal complexes. For example, recently, tetrahedral carbonyl hydride cluster $\mathrm{H}_{4} \mathrm{Ru}_{4}(\mathrm{CO})_{11} \mathrm{~L}$ have been synthesized using the ligand (L) di(2-phenethyl)[2-(octahydro- $2 \mathrm{H}$ quinolizin-1-ylmethyloxy)ethyl]phosphine $6^{15}$. The structure and dynamic behavior of the cluster in solution have been studied. 


\section{Experimental Section}

General Procedures. ${ }^{1} \mathrm{H},{ }^{13} \mathrm{C},{ }^{31} \mathrm{P}$ and ${ }^{77} \mathrm{Se}$ NMR spectra were recorded on Bruker DPX-400 (400.13, 100.61, 161.98 and $76.26 \mathrm{MHz}$, respectively) spectrometer in $\mathrm{CDCl}_{3}$ solutions and referenced to internal HMDS $\left({ }^{1} \mathrm{H}\right), \mathrm{CHCl}_{3}\left({ }^{13} \mathrm{C}\right)$ and external $85 \% \mathrm{H}_{3} \mathrm{PO}_{4}\left({ }^{31} \mathrm{P}\right)$ and $\mathrm{Me}_{2} \mathrm{Se}\left({ }^{77} \mathrm{Se}\right)$. IR spectra were measured with a Bruker IFS 25 instrument in microlayer. Optical rotation measurements were carried out on a Polamat A polarimeter.

General procedure for the synthesis of tertiary diorganyl[2-(octahydro- $2 \mathrm{H}$-quinolizin-1ylmethyloxy)ethyl]phosphines 5, 6 and -phosphine sulfide 7 (Table 1)

An equimolar mixture of secondary phosphine 2, 3 or phosphine sulfide 4 and vinyl ether of lupinine 1 was heated at $65-70{ }^{\circ} \mathrm{C}$ in the presence of AIBN (0.5-1.5 wt $\%$ of the reactants' mass) in a sealed ampoule. The reaction was monitored by ${ }^{31} \mathrm{P}$ NMR spectroscopy. The crude product, a viscous undistillable liquid, was purified by column chromatography on $\mathrm{Al}_{2} \mathrm{O}_{3}$ (diethyl ether) to give the phosphine 5, 6 or phosphine sulfide 7. All operations were scrupulously carried out under an atmosphere of inert gas (preferably, argon).

Dibutyl[2-(octahydro-2H-quinolizin-1-ylmethyloxy)ethyl]phosphine (5). Yellowish oil, $0.8 \mathrm{~g}$ (93\%) yield, $[\alpha]^{22}{ }_{\mathrm{D}}-12.0(\mathrm{c} 2.0, \mathrm{EtOH}) .{ }^{1} \mathrm{H} \mathrm{NMR}, \delta(\mathrm{ppm}), J(\mathrm{~Hz}): 0.84\left(\mathrm{t},{ }^{3} J=6.8,6 \mathrm{H}, \mathrm{Me}\right)$, 1.16 (m, 1H, H-8'), 1.28-1.39 (m, 14H, H-2,3,9, $\left.\underline{\mathrm{CH}}_{2} \mathrm{C}_{2} \mathrm{Me}\right), 1.49$ (m, 2H, H-7), 1.62-1.75 (m, 8H, H-1,8",13, $\left.\mathrm{CH}_{2} \mathrm{P}\right), 1.84-1.93$ (m, 3H, H-4a,6a,9a), 2.75 (m, 2H, H-4e,6e), 3.39-3.54 (m, 2H, $\mathrm{H}-12), 3.44\left(\mathrm{dd},{ }^{2} J=9.3,{ }^{3} J=5.5,1 \mathrm{H}, \mathrm{H}-10^{\prime}\right)$ and $3.58\left(\mathrm{dd},{ }^{3} J=7.0 \mathrm{~Hz}, 1 \mathrm{H}, \mathrm{H}-10 "\right) .{ }^{13} \mathrm{C} \mathrm{NMR}, \delta$ (ppm), $J(\mathrm{~Hz}): 13.76(\mathrm{Me}), 21.20(\mathrm{C}-3), 24.41\left(\mathrm{~d},{ }^{2} J_{\mathrm{PC}}=11.1, \underline{\mathrm{CH}}_{2} \mathrm{C}_{2} \mathrm{H}_{5}\right), 25.03\left(\mathrm{C}-8, \underline{\left.\mathrm{CH}_{2} \mathrm{Me}\right),}\right.$ $25.48(\mathrm{C}-7), 26.97\left(\mathrm{~d},{ }^{1} J_{\mathrm{PC}}=11.5, \mathrm{PCH}_{2}\right), 27.09(\mathrm{C}-9), 27.73\left(\mathrm{~d},{ }^{1} J_{\mathrm{PC}}=13.7, \mathrm{C}-13\right), 27.86(\mathrm{~d}$, $\left.{ }^{1} J_{\mathrm{PC}}=11.8, \mathrm{PCH}_{2}\right), 29.74(\mathrm{C}-2), 38.65(\mathrm{C}-1), 57.42(\mathrm{C}-4,6), 64.70(\mathrm{C}-9 \mathrm{a}), 68.70\left(\mathrm{~d},{ }^{2} J_{\mathrm{PC}}=20.3\right.$, C-12), 69.35 (C-10). ${ }^{31} \mathrm{P}$ NMR, $\delta$ (ppm): -33.91. Anal. Calcd. for $\mathrm{C}_{20} \mathrm{H}_{40} \mathrm{NOP}: \mathrm{C}, 70.34 ; \mathrm{H}$, 11.81 ; N, 4.10; P, 9.07\%. Found: C, 70.15; H, 11.70; N, 4.00; P, 9.20\%.

Di(2-phenethyl)[2-(octahydro-2H-quinolizin-1-ylmethyloxy)ethyl]phosphine (6). Yellowish oil, $1.2 \mathrm{~g}(89 \%)$ yield, $[\alpha]^{22} \mathrm{D}-9.0$ (c 1.0, EtOH). ${ }^{1} \mathrm{H}$ NMR, $\delta(\mathrm{ppm}): 1.32-1.37$ (m, 1H, H-8'), 1.42-1.70 (m, 6H, H-2,3,7), 1.80-1.93 (m, 9H, H-1,4a,6a,8",9,9a,13), 2.02-2.09 (m, 4H, PC $\left.\underline{H}_{2}\right)$, 2.84-2.93 (m, 6H, H-4e,6e, $\left.\underline{\mathrm{C}}_{2} \mathrm{Ph}\right), 3.49-3.74$ (m, 4H, H-10,12), 7.29-7.42 (m, $\left.10 \mathrm{H}, \mathrm{Ph}\right) .{ }^{13} \mathrm{C}$ NMR, $\delta(\mathrm{ppm}), J(\mathrm{~Hz}): 21.23$ (C-3), 25.01 (C-8), 25.45 (C-7), 27.09 (C-9), 27.58 (d, ${ }^{1} J_{\mathrm{PC}}=14.6$, C-13), $29.31\left(\mathrm{~d},{ }^{1} J_{\mathrm{PC}}=13.3, \underline{\mathrm{PCH}}_{2}\right), 29.81(\mathrm{C}-2), 32.21\left(\mathrm{~d},{ }^{2} J_{\mathrm{PC}}=14.6, \underline{\mathrm{CH}}_{2} \mathrm{Ph}\right), 38.65(\mathrm{C}-1)$, $57.40(\mathrm{C}-4,6), 64.66(\mathrm{C}-9 \mathrm{a}), 68.63\left(\mathrm{~d},{ }^{2} J_{\mathrm{PC}}=19.3, \mathrm{C}-12\right), 69.59$ (C-10), 125.89 (C-p), 128.06 (C$o), 128.39(\mathrm{C}-m), 142.84\left(d,{ }^{3} J_{\mathrm{PC}}=10.7, \mathrm{C}-i\right) .{ }^{31} \mathrm{P} \mathrm{NMR}, \delta(\mathrm{ppm}):-30.03$. Anal. Calcd. for $\mathrm{C}_{28} \mathrm{H}_{40} \mathrm{NOP}$ : C, 76.85; H, 9.21; N, 3.20; P, 7.08\%. Found: 76.65; H, 9.40; N, 3.12; P, 6.98\%.

Dibutyl[2-(octahydro-2H-quinolizin-1-ylmethyloxy)ethyl]phosphine sulfide (7). Yellowish oil, $0.46 \mathrm{~g}(96 \%)$ yield, $[\alpha]^{22} \mathrm{D}-6.6$ (c 1.5, EtOH); ${ }^{1} \mathrm{H}$ NMR, $\delta(\mathrm{ppm}), J(\mathrm{~Hz}): 0.92\left(\mathrm{t},{ }^{3} J=7.2\right.$, $6 \mathrm{H}, \mathrm{Me}), 1.24$ (m, 1H, H-8'), 1.39 (m, 4H, $\left.\mathrm{CH}_{2} \mathrm{Me}\right), 1.38-1.47$ (m, 4H, H-7,9), 1.53-1.60 (m, 6H, H-3, $\underline{\mathrm{C}}_{2} \mathrm{C}_{2} \mathrm{H}_{5}$ ), 1.71-1.89 (m, 9H, H-1,2,8",9a, $\mathrm{PC}_{2}$ ), 2.07 (m, 2H, H-4a,6a), 2.09 (m, 2H, H13), 2.91 (m, 2H, H-4e,6e), 3.50-3.80 (m, 4H, H-10,12). ${ }^{13} \mathrm{C} \mathrm{NMR}, \delta(\mathrm{ppm}), J(\mathrm{~Hz}): 13.46(\mathrm{Me})$, 
$21.02(\mathrm{C}-3), 23.51(\mathrm{C}-9), 23.70$ and $23.85\left(\underline{\mathrm{CH}}_{2} \mathrm{Me}\right), 24.26(\mathrm{C}-8), 24.28\left(\mathrm{~d},{ }^{2} J_{\mathrm{PC}}=7.3, \underline{\mathrm{CH}}_{2} \mathrm{C}_{2} \mathrm{H}_{5}\right)$, $24.46(\mathrm{C}-2,7), 30.96\left(\mathrm{~d},{ }^{1} J_{\mathrm{PC}}=49.8, \mathrm{C}-13\right), 31.12$ and $31.45\left(\right.$ two d, $\left.{ }^{1} J_{\mathrm{PC}}=50.5, \mathrm{PCH}_{2}\right), 38.15(\mathrm{C}-$ 1), 56.70 (C-4,6), 64.06 (C-9a), 64.74 (d, $\left.{ }^{2} J_{\mathrm{PC}}=3.0, \mathrm{C}-12\right), 69.90$ (C-10). ${ }^{31} \mathrm{P}$ NMR, $\delta(\mathrm{ppm})$ : 48.41. IR, $v\left(\mathrm{~cm}^{-1}\right)$ : 1010 (C-O-C), $594(\mathrm{P}=\mathrm{S})$. Anal. Calcd. for $\mathrm{C}_{20} \mathrm{H}_{40} \mathrm{NOPS}$ : C, 64.30; H, 10.79; N, 3.75; P, 8.29, S, 8.58\%. Found: C, 64.42; H, 10.80; N, 3.80; P, 7.98; S, 8.80\%.

\section{General procedure for the synthesis of tertiary phosphine oxides 8, 9 (Table 2)}

A solution of phosphine 5, 6 in hexane was stirred at r.t. under air atmosphere. The reaction was monitored by ${ }^{31} \mathrm{P}$ NMR spectroscopy. After completion of the reaction the solvent was evaporated under reduced pressure to afford phosphine oxide $\mathbf{8 , 9}$.

Dibutyl[2-(octahydro-2H-quinolizin-1-ylmethyloxy)ethyl]phosphine oxide (8). Colorless oil, $0.15 \mathrm{~g}(95 \%)$ yield, $[\alpha]^{24} \mathrm{D}-5.9(c 0.5, \mathrm{EtOH}) .{ }^{1} \mathrm{H} \mathrm{NMR}, \delta(\mathrm{ppm}), J(\mathrm{~Hz}): 0.84$ and 0.85 (two t, $\left.{ }^{3} J_{\mathrm{HH}}=7.2,6 \mathrm{H}, \mathrm{Me}\right), 1.12-1.17\left(\mathrm{~m}, 2 \mathrm{H}, \mathrm{H}-8^{\prime}, 9^{\prime}\right), 1.33-1.36\left(\mathrm{~m}, 4 \mathrm{H}, \mathrm{C}_{2} \mathrm{Me}\right), 1.39-1.60$ (m, 9H, $\mathrm{H}-2,7,9 ", \mathrm{CH}_{2} \mathrm{C}_{2} \mathrm{H}_{5}$ ), 1.60-1.75 (m, 9H, H-1,3,8",9a, $\mathrm{PC}_{2}$ ), 1.90-1.98 (m, 2H, H-4a,6a), 2.082.10 (m, 2H, H-13), 2.86-2.88 (m, 2H, H-4e,6e), 3.54-3.70 (m, 4H, H-10,12). ${ }^{13} \mathrm{C}$ NMR, $\delta$ (ppm), $J(\mathrm{~Hz}): 13.59(\mathrm{Me}), 21.01(\mathrm{C}-3), 23.63$ and 23.66 (two d, ${ }^{3} J_{\mathrm{PC}}=3.8, \underline{\mathrm{CH}}_{2} \mathrm{Me}$ ), 24.21 (d, $\left.{ }^{2} J_{\mathrm{PC}}=14.5, \underline{\mathrm{CH}}_{2} \mathrm{C}_{2} \mathrm{H}_{5}\right), 24.26(\mathrm{C}-8), 24.40$ (C-7), 26.50 (C-2), 27.09 (C-9), 28.38 and 28.44 (d, ${ }^{1} J_{\mathrm{PC}}=64.8$ and 65.2, $\left.\underline{\mathrm{PCH}}_{2}\right), 28.53\left(\mathrm{~d},{ }^{1} J_{\mathrm{PC}}=63.4, \mathrm{C}-13\right), 38.03(\mathrm{C}-1), 56.79(\mathrm{C}-4,6), 64.44(\mathrm{~d}$, $\left.{ }^{2} J_{\mathrm{PC}}=2.7, \mathrm{C}-12\right), 64.68(\mathrm{C}-9 \mathrm{a}), 70.12$ (C-10). ${ }^{31} \mathrm{P} \mathrm{NMR}, \delta(\mathrm{ppm}): 48.63 . \mathrm{IR}, v\left(\mathrm{~cm}^{-1}\right): 1065$ $(\mathrm{P}=\mathrm{O}), 1010$ (C-O-C). Anal. Calcd. for $\mathrm{C}_{20} \mathrm{H}_{40} \mathrm{NO}_{2} \mathrm{P}: \mathrm{C}, 67.19 ; \mathrm{H}, 11.28 ; \mathrm{N}, 3.92 ; \mathrm{P}, 8.66 \%$. Found: C, 67.48; H, 11.08; N, 3.65; P, 8.36\%.

Di(2-phenethyl)[2-(octahydro-2H-quinolizin-1-ylmethyloxy)ethyl]phosphine oxide (9). Colorless oil, $0.15 \mathrm{~g}(97 \%)$ yield, $[\alpha]^{20}{ }_{\mathrm{D}}-1.6\left(c\right.$ 1.5, $\left.\mathrm{CHCl}_{3}\right) .{ }^{1} \mathrm{H} \mathrm{NMR}, \delta(\mathrm{ppm}): 1.15-1.25(\mathrm{~m}$, 1H, H-8'), 1.27-1.44 (m, 4H, H-2,3), 1.47-1.53 (m, 2H, H-7), 1.62-1.71 (m, 2H, H-1,8"), 1.721.80 (m, 5H, H-4a,6a,9,9a), 1.90-2.08 (m, 6H, H-13, $\mathrm{PC}_{2}$ ), 2.69-2.82 (m, 6H, H-4e, $6 e, \underline{\mathrm{C}}_{2} \mathrm{Ph}$ ), 3.49-3.65 (m, 4H, H-10,12), 7.12-7.28 (m, 10H, Ph). ${ }^{13} \mathrm{C} \mathrm{NMR,} \delta$ (ppm), $J(\mathrm{~Hz}): 21.06(\mathrm{C}-3)$, $24.85(\mathrm{C}-8), 25.30$ (C-7), $27.00(\mathrm{C}-9), 27.58\left(\mathrm{~d},{ }^{2} J_{\mathrm{PC}}=17.6, \underline{\mathrm{CH}}_{2} \mathrm{Ph}\right), 28.89\left(\mathrm{~d},{ }^{1} J_{\mathrm{PC}}=62.3, \mathrm{C}-\right.$ 13), $29.57(\mathrm{C}-2), 32.21\left(\mathrm{~d},{ }^{1} J_{\mathrm{PC}}=63.0, \mathrm{PCH}_{2}\right), 38.47(\mathrm{C}-1), 57.21(\mathrm{C}-4,6), 63.93\left(\mathrm{~d},{ }^{2} J_{\mathrm{PC}}=3.9\right.$, C-12), 64.46 (C-9a), 69.40 (C-10), 125.66 (C-p), $127.83(\mathrm{C}-o), 128.15(\mathrm{C}-m), 141.58\left(\mathrm{~d},{ }^{3} J_{\mathrm{PC}}=\right.$ 13.0, C-i). ${ }^{31} \mathrm{P}$ NMR, $\delta$ (ppm): 46.43. IR, $v\left(\mathrm{~cm}^{-1}\right): 1158(\mathrm{P}=\mathrm{O}), 1108,1095$ (C-O-C). Anal. Calcd. for $\mathrm{C}_{28} \mathrm{H}_{40} \mathrm{NO}_{2} \mathrm{P}: \mathrm{C}$, 74.14; H, 8.89; N, 3.09; P, 6.83\%. Found: C, 74.28; H, 9.08; N, 3.15; $\mathrm{P}, 6.56 \%$.

\section{General procedure for the synthesis of tertiary phosphine sulfides 7, 10 (Table 2)}

A mixture of phosphine 2, 3 and elemental sulfur in toluene was heated at $50{ }^{\circ} \mathrm{C}$ upon stirring under argon for $3 \mathrm{~h}$. Unreacted $\mathrm{S}_{8}$ was filtered and the solvent was removed from the filtrate. The crude product, a viscous undistillable liquid, was purified by column chromatography on $\mathrm{Al}_{2} \mathrm{O}_{3}$ (hexane) to give the phosphine sulfide $7, \mathbf{1 0}$.

Dibutyl[2-(octahydro-2H-quinolizin-1-ylmethyloxy)ethyl]phosphine sulfide (7). Yellowish oil, $0.29 \mathrm{~g}(88 \%)$ yield. 
Di(2-phenethyl)[2-(octahydro-2H-quinolizin-1-ylmethyloxy)ethyl]phosphine sulfide (10). Yellowish oil, $0.29 \mathrm{~g}(90 \%)$ yield, $[\alpha]^{23} \mathrm{D}-6.2$ (c 2.0, EtOH). ${ }^{1} \mathrm{H}$ NMR, $\delta(\mathrm{ppm}): 1.30$ (m, 2H, H$\left.2^{\prime}, 8^{\prime}\right)$, 1.38-1.42 (m, 3H, H-2",3), 1.48-1.52 (m, 2H, H-7), 1.60-1.80 (m, 3H, H-1,8",9'), 1.88-1.92 (m, 4H, H-4a,6a,9",9a), 2.11-2.20 (m, 6H, H-13, PC 2 ), 2.79 (m, 2H, H-4e,6e), 2.91-2.94 (m, $\left.4 \mathrm{H}, \mathrm{CH}_{2} \mathrm{Ph}\right), 3.59-3.79(\mathrm{~m}, 4 \mathrm{H}, \mathrm{H}-10,12), 7.18-7.27(\mathrm{~m}, 10 \mathrm{H}, \mathrm{Ph}) .{ }^{13} \mathrm{C} \mathrm{NMR}, \delta(\mathrm{ppm}), J(\mathrm{~Hz})$ : 21.27 (C-3), 24.99 (C-8), $25.33(\mathrm{C}-7), 27.16(\mathrm{C}-9), 28.60\left(\underline{\mathrm{CH}}_{2} \mathrm{Ph}\right), 29.72(\mathrm{C}-2), 31.65\left(\mathrm{~d},{ }^{1} J_{\mathrm{PC}}=\right.$ 49.5, C-13), 33.50 and 33.54 (two d, ${ }^{1} J_{\mathrm{PC}}=48.0$ and 48.4, $\underline{\mathrm{PCH}}_{2}$ ), $38.58(\mathrm{C}-1), 57.33(\mathrm{C}-4,6)$, $64.50(\mathrm{C}-9 \mathrm{a}), 64.86\left(\mathrm{~d},{ }^{2} J_{\mathrm{PC}}=4.0, \mathrm{C}-12\right), 70.31(\mathrm{C}-10), 126.47(\mathrm{C}-p), 128.25(\mathrm{C}-o), 128.68(\mathrm{C}-$ $m), 140.86\left(\mathrm{~d},{ }^{3} J_{\mathrm{PC}}=14.3, \mathrm{C}-i\right) .{ }^{31} \mathrm{P}$ NMR, $\delta(\mathrm{ppm}): 48.11 . \mathrm{IR}, v\left(\mathrm{~cm}^{-1}\right): 1101(\mathrm{C}-\mathrm{O}-\mathrm{C}), 601$ $(\mathrm{P}=\mathrm{S})$. Anal. Calcd. for $\mathrm{C}_{28} \mathrm{H}_{40}$ NOPS: C, 71.60; H, 8.58; N, 2.98; P, 6.59; S, 6.83\%. Found: C, 71.30; H, 8.50; N, 3.05; P, 6.25; S, 6.95\%.

\section{Synthesis of di(2-phenethyl)[2-(octahydro-2H-quinolizin-1-ylmethyloxy)ethyl]-phosphine selenide (11)}

A mixture of phosphine $3(0.33 \mathrm{~g}, 0.75 \mathrm{mmol})$ and elemental selenium $(0.07 \mathrm{~g}, 0.89 \mathrm{mmol})$ in toluene was heated at $50{ }^{\circ} \mathrm{C}$ upon stirring under argon for $3 \mathrm{~h}$. Unreacted Se was filtered and the solvent was removed from the filtrate. The crude product, a viscous undistillable liquid, was purified by column chromatography on $\mathrm{Al}_{2} \mathrm{O}_{3}$ (hexane).Yellowish oil, $0.29 \mathrm{~g}(75 \%)$ yield, $[\alpha]^{22}{ }_{\mathrm{D}}$ -8.9 (c 2.0, $\left.\mathrm{CHCl}_{3}\right) .{ }^{1} \mathrm{H} \mathrm{NMR}, \delta$ (ppm): 1.20-1.25 (m, 2H, H-2', $\left.8^{\prime}\right), 1.39$ (m, 3H, H-2",3), 1.50 1.59 (m, 2H, H-7), 1.60-1.80 (m, 4H, H-1,8",9), 1.90 (m, 3H, H-4a,6a,9a), 2.21-2.30 (m, 6H, H13, $\left.\mathrm{PC}_{2}\right), 2.79$ (m, 2H, H-4e,6e), 2.80-2.98 (m, 4H, $\left.\mathrm{C}_{2} \mathrm{Ph}\right), 3.59-3.79$ (m, 4H, H-10,12), 7.18$7.27(\mathrm{~m}, 10 \mathrm{H}, \mathrm{Ph}) .{ }^{13} \mathrm{C}$ NMR, $\delta(\mathrm{ppm}), J(\mathrm{~Hz}): 21.28(\mathrm{C}-3), 24.97(\mathrm{C}-8), 27.09(\mathrm{C}-7), 27.75(\mathrm{C}-$ 9), $29.41\left(\underline{\mathrm{CH}}_{2} \mathrm{Ph}\right), 29.73(\mathrm{C}-2), 31.51\left(\mathrm{~d},{ }^{1} J_{\mathrm{PC}}=43.6, \mathrm{C}-13\right), 32.62$ and 33.11 (two d, ${ }^{2} J_{\mathrm{PC}}=41.0$, $\left.\mathrm{PCH}_{2}\right), 38.58(\mathrm{C}-1), 57.33(\mathrm{C}-4,6), 64.37(\mathrm{C}-9 \mathrm{a}), 65.57\left(\mathrm{~d},{ }^{2} J_{\mathrm{PC}}=3.7, \mathrm{C}-12\right), 69.79(\mathrm{C}-10)$, $126.54(\mathrm{C}-p), 128.32(\mathrm{C}-o), 128.72(\mathrm{C}-m), 140.63\left(\mathrm{~d},{ }^{3} J_{\mathrm{PC}}=14.7, \mathrm{C}-i\right) .{ }^{31} \mathrm{P} \mathrm{NMR}, \delta(\mathrm{ppm}): 40.68$. ${ }^{77} \mathrm{Se}$ NMR, $\delta(\mathrm{ppm}), J(\mathrm{~Hz}):-384.89\left(\mathrm{~d},{ }^{1} J_{\mathrm{PSe}} 696.1\right) . \mathrm{IR}, v\left(\mathrm{~cm}^{-1}\right): 1101(\mathrm{C}-\mathrm{O}-\mathrm{C}), 464(\mathrm{P}=\mathrm{Se})$. Anal. Calcd. for $\mathrm{C}_{28} \mathrm{H}_{40} \mathrm{NOPSe}$ C, 65.10; H, 7.81; N, 2.41; P, 6.00; Se, 15.29\%. Found: C, 64.90; H, 7.60; N, 2.15; P, 6.25; Se, 14.95\%.

\section{Acknowledgements}

Financial support from the Russian Foundation for Basic Research (grant no. 07-03-00562) is gratefully acknowledged.

\section{References}

1. (a) Michael, J. P. In The Alkaloids, Chemistry and Pharmacology; Cordell, G.; Academic Press: New York, 2001, pp 91-258. (b) Lourenço, A. M.; Máximo, P.; Ferreira, L. M.; 
Pereira, M. M. A. In Studies in Natural Product Chemistry. Bioactive Natural Products. Part H; Atta-ur-Rahman; Elsevier: Amsterdam, 2002; pp 233 - 298.

2. Daly, J. W.; Carraffo, H. M.; Spande, T. F. Alkaloids: Chemical and Biological Perspectives Vol. 13, Ch. 1; Pelletier, S. W.; Wiley: New York, 1999.

3. Cho, J.; Kim, D. Chem. Rev. 2005, 105, 4779.

4. Ding, P.-L.; Uuang, H.; Zhou, P.; Chem, D.-F. Planta Medica 2006, 72, 854.

5. (a) El-Shazly, A.; Ateya, A.-M. M.; Wink, M. Z. Naturforsch 2001, 56c, 21. (b) Kikuchi, H.; Tasaka, H.; Hirai, S.; Takaya, Y.; Iwabuchi, Y.; Ooi, H.; Hatakeyama, S.; Kim, H.-S.; Wataya, Y.; Oshima, Y. J. Med. Chem. 2002, 45, 2563. (c) Tlegenov, R. T. Khim. Rast. Syrya 2007, no. 1, 49 (in Russian); (d) Tlegenov, R. T. Khim. Rast. Syrya 2007, 4, 69 (in Russian).

6. (a) Yu, S.; Pu, X.; Cheng, T.; Wang, R.; Ma, D. Org. Lett. 2006, 8, 3179; (b) LaroucheGauthier, R.; Bélanger, G. Org. Lett. 2008, 10, 4501; (c) Ercoli, M.; Mina, L.; Canu Boido, C.; Boido, V.; Sparatore, F.; Armani, U.; Piana, A. Farmaco 2004, 59, 101.

7. (a) Michael, J. P. Beilstein J. Org. Chem. 2007, 3. doi:10.1186/1860-5397-3-27. (b) Michael, J. P. Nat. Prod. Rep. 2008, 25, 131; (c) Remuson, R. Beilstein J. Org. Chem. 2007, 3. doi:10.1186/1860-5397-3-32.

8. $\quad$ Sparatore, A.; Novelli, F.; Sparatore, F. Helv. Chim. Acta 2004, 87, 580.

9. (a) Schwink, L.; Stengelin, S.; Gossel, M.; Hessler, G.; Lennig, P. Pat. Appl. WO 2006018279, 2006. (b) Orlek, B. S. Pat. Appl. WO 9503302, 1995.

10. (a) Chakravarty, P. K.; Colletti, S. L.; Ingenito, R.; Jones, P.; Meinke, P. T.; Petrocchi, A.; Steinkuhler, C. Pat. Appl. WO 2006005955, 2006. (b) Jones P. Pat. Appl. WO 20090054448, 2009.

11. (a) Arbuzova, S. N.; Brandsma, L.; Gusarova, N. K.; Trofimov, B. A. Recl. Trav. Chim. Pays-Bas. 1994, 113, 575. (b) Trofimov, B. A.; Brandsma, L.; Arbuzova, S. N.; Malysheva, S. F.; Gusarova, N. K. Tetrahedron Lett. 1994, 35, 7647.

12. Gusarova, N. K.; Bogdanova, M. V.; Ivanova, N. I.; Chernysheva, N. A.; Sukhov, B. G.; Sinegovskaya, L. M.; Kazheva, O. N.; Alexandrov, G. G.; D’yachenko, O. A.; Trofimov, B. A. Synthesis 2005, 3103.

13. Oparina, L. A.; Tlegenov, R. T.; Ermakova, T. G.; Kuznetsova, N. P.; Kanitskaya, L. V.; Tantsyrev, A. P.; Trofimov, B. A. Russ. Chem. Bull. Int. Ed. 2004, 53, 232.

14. (a) Malysheva, S. F.; Gusarova, N. K., Belogorlova, N. A.; Nikitin, M. V.; Gendin, D. V. Russ. J. Gen. Chem. 1997, 67, 58. (b) Trofimov, B. A.; Gusarova, N. K.; Malysheva, S. F.; Ivanova, N. I.; Sukhov, B. G.; Belogorlova, N. A.; Kuimov, V. A. Synthesis 2002, 2207. (c) Gusarova, N. K.; Ivanova, N. I.; Bogdanova, M. V.; Malysheva, S. F.; Belogorlova, N. A.; Sukhov, B. G.; Trofimov, B. A. Mend. Commun. 2004, 216. (d) Trofimov, B. A.; Sukhov, B. G.; Malysheva, S. F.; Belogorlova, N. A.; Tantsirev, A. P.; Parshina, L. N.; Oparina, L. A.; Tunik, S. P.; Gusarova, N. K. Tetrahedron Lett. 2004, 45, 9143. (e) Parsons, A. F.; Sharpe, D. J.; Taylor, Ph. Synlett. 2005, 2981. (f) Hirai, T.; Han, L.-B. Org. Lett. 2007, 9, 53. 
15. Andreev, I. A.; Gracheva, E. V.; Tunik, S. P.; Oparina, L. A.; Sukhov, B. G.; Malysheva, S. F.; Kinoshita, I.; Nishioka, T. Russ. Chem Bull. Int. Ed. 2007, 56, 1343. 